\title{
A SPACE OF SUBSETS HAVING THE FIXED POINT PROPERTY ${ }^{1}$
}

\author{
C. E. CAPEL AND W. L. STROTHER
}

In 1939 Wojdyslawski [6] asked whether the property of being a $\mathrm{CAR}^{*}$ (= retract of a Tychonoff cube) is preserved from a space $X$ to the space $S(X)$ of non-null closed subsets of $X$. This has been answered affirmatively for a special case only, namely, when $X$ is a Peano space. Whether the fixed point property is preserved from $X$ to $S(X)$ is also unknown. Both properties fail to be preserved in the opposite direction [4, Corollary 1 and Corollary 10].

The object of this paper is to prove that if $X$ is a CAR* then $S(X)$ has the fixed point property. First it will be shown that if $T$ is a Tychonoff cube then $S(T)$ has the fixed point property. If the space $X$ is a CAR* $^{*}$ it is a retract of some cube $T$ and hence [4] $S(X)$ is a retract of $S(T)$. Thus the fixed point property in $S(X)$ follows from that in $S(T)$.

The following notation will be used. If $Y$ is a space then $S(Y)$ denotes the set of non-null closed subsets of $Y$ with the usual topology [5, p. 281]. For each element $a$ in a set $A$ let $I_{a}$ be the unit interval; then the cartesian product $T=P\left\{I_{a}: a \in A\right\}$ is a Tychonoff cube. Let $B=\{B: B$ is a finite, non-null subset of $A\}$; then for each $B$ in $B$ let $T_{B}=P\left\{I_{a}: a \in B\right\}$. The projection functions are given by $\pi_{a}: T \rightarrow I_{a}$ and $\pi_{B}: T \rightarrow T_{B}$.

The proof of the theorem depends upon a collection of subsets of $S(T)$ defined, for $B$ in $B$, by $S(T, B)=S\left(T_{B}\right) \times P\left\{S\left(I_{a}\right): a \notin B\right\}$. The function $r_{B}: S(T) \rightarrow S(T, B)$, defined by $r_{B}(E)=\pi_{B}(E) \times P\left\{\pi_{a}(E)\right.$ : $a \notin B\}$, is a retraction of $S(T)$ onto $S(T, B)[1$, p. 68].

The fact that the system $(B, C)$ is a directed set is used in the following lemma. For details on nets see [2].

Lemma. If $\mathfrak{C}$ is a cofinal subset of $\mathbb{B}$ and $\left\{E_{C}\right\}$ is a net in $S(T)$ on e then lime $\left\{E_{C}\right\}=E$ implies lime $\left\{r_{C}\left(E_{C}\right)\right\}=E$.

Proof. Let $N(W ; V)$ be a subbasic element of the topology of $S(T)$ such that $E \in N(W ; V)$. It is sufficient to show that the net $\left\{r_{C}\left(E_{C}\right)\right\}$ is eventually in $N(W ; V)$. Now $E$ being in $N(W ; V)$ means that $E \subset W$ and $E \cap V \neq 0$ where $W$ and $V$ are open subsets of $T$.

Presented to the Society, September 2, 1955 under the title Fixed point property for the space of subsets of an absolute retract; received by the editors August 9, 1955.

1 This research was supported by the United States Air Force, through the Office of Scientific Research of the Air Research and Development Command. 
Since $E$ is compact we may assume $W=\bigcup\left\{W_{i}: i=1, \cdots, n\right\}$, a finite union of basis elements of the form $W_{i}=P\left\{U_{a}: a \in B_{i}\right\}$ $\times P\left\{I_{a}: a \notin B_{i}\right\}$ where $U_{a}$ is an open subset of $I_{a}$. Let $B_{0}=\bigcup\left\{B_{i}\right.$ : $i=1, \cdots, n\}$.

If lime $\left\{E_{C}\right\}=E$ then there is $C_{1}$ in $\mathfrak{e}$ such that $E_{C} \in N(W ; V)$ whenever $C$ contains $C_{1}$. Let $C_{2}$ be in $\mathcal{e}$ such that $C_{1} \subset C_{2}$ and $B_{0} \subset C_{2}$.

Let $C$ be any element of $\mathcal{C}$ which contains $C_{2}$. If $x \in r_{C}\left(E_{C}\right)$ then there is $y \in E_{C}$ such that $\pi_{C}(x)=\pi_{C}(y)$. Now $y \in E_{C} \subset W$ and therefore for some $i, y \in W_{i}$. Since $B_{0} \subset C_{2}$, it follows that $x \in W_{i}$ and hence $r_{C}\left(E_{C}\right) \subset W$. Note that $E_{C} \subset r_{C}\left(E_{C}\right)$ so that $E_{C} \cap V \neq 0$ implies $r_{C}\left(E_{C}\right)$ $\cap V \neq 0$. This means that $r_{C}\left(E_{C}\right) \in N(W ; V)$ whenever $C$ contains $C_{2}$, which completes the proof.

Theorem. The space $S(T)$ has the fixed point property.

Proof. It is known [3, p. 117] that $S(T)$ can be imbedded in some Tychonoff cube $R$ and we identify $S(T)$ with its homeomorphic image in $R$. Since the spaces $T_{B}$ and $I_{a}$ are all Peano spaces, it follows [4, Theorem 8] that $S\left(T_{B}\right)$ and $S\left(I_{a}\right)$ are CAR*'s, and hence each $S(T, B)$ is a CAR*. Each retraction $r_{B}: S(T) \rightarrow S(T, B)$ can therefore be extended to a retraction $p_{B}: R \rightarrow S(T, B)$.

Now let $f: S(T) \rightarrow S(T)$ be a continuous function. The composition $f p_{B}: R \rightarrow S(T) \subset R$ is a continuous function on a cube into itself and has a fixed point. Let $E_{B}$ be a fixed point of $f p_{B}$; then $E_{B} \in S(T)$. This determines a net $\left\{E_{B}\right\}$ in the compact space $S(T)$ on $B$ and hence [2, Theorem 24] there exists a subnet $\left\{E_{C}\right\}$ on a cofinal subset $\mathfrak{C}$ of $B$ with a limit $E$ in $S(T)$. By the lemma lime $\left\{E_{C}\right\}=E$ implies lime $\left\{r_{C}\left(E_{C}\right)\right\}=E$. Since $f$ is continuous this means that lime $\left\{f r_{C}\left(E_{C}\right)\right\}=f(E)$ but $f r_{C}\left(E_{C}\right)=f p_{C}\left(E_{C}\right)=E_{C}$. Hence $f(E)=E$ and the theorem is proved.

Corollary. If $X$ is a $C A R^{*}$ then $S(X)$ has the fixed point property.

\section{REFERENCES}

1. C. E. Capel, Space of subsets and cartesian products, Bull. Amer. Math. Soc. Abstract 61-1-117.

2. J. L. Kelley, Convergence in topology, Duke Math. J. vol. 17 (1950) pp. 277-283.

3. - General topology, New York, Van Nostrand, 1955.

4. W. L. Strother, Fixed points, fixed sets, and M-retracts, Duke Math. J. vol. 22 (1955) pp. 551-556.

5. —, Multi-homotopy, Duke Math. J. vol. 22 (1955) pp. 281-286.

6. M. Wojdyslawski, Retractes absolus et hyperespaces des continus, Fund. Math. vol. 32 (1939) pp. 184-192.

UNIVERSITY OF MiAMI 